\title{
A New Biological Strategy for Drug Delivery: Eucaryotic Cell-Derived Nanovesicles
}

\author{
Irène Tatischeff $^{1 *}$, Annette Alfsen ${ }^{2}$ \\ ${ }^{1}$ Laboratoire Acides Nucléiques et Biophotonique (ANBioPhi), Université Pierre et Marie Curie, Paris, France; ${ }^{2}$ Institut Cochin, Dé- \\ partement de Biologie Cellulaire, Université Paris-Descartes, Paris, France. \\ E-mail: *irene.tatischeff@upmc.fr
}

Received October $4^{\text {th }}, 2011$; revised November $13^{\text {th }}, 2011$; accepted November $25^{\text {th }}, 2011$.

\begin{abstract}
An efficient drug delivery is the prerequisite of the successful chemotherapeutic treatments of many human diseases. Despite a great number of approaches, the improvement of drug cell internalization remains an actual research challenge. We propose a new biological delivery system based on the extracellular vesicles released by a non-pathological eukaryotic microorganism, Dictyostelium discoideum. After a summary of the main characteristics of these extracellular vesicles, including of their lipid bilayer that appears as a good candidate for initiating membrane fusion, followed by delivery of their encapsulated drug, the capacity of these vesicles to convey drugs into human cells was demonstrated in vitro on two tumor cell lines, resistant leukaemia K562r and cervix carcinoma HeLa cells. A comparison with other extracellular vesicles, like exosomes or bacteria-derived particles, stresses the unique properties of Dictyostelium extracellular nanovesicles for drug delivery.
\end{abstract}

Keywords: Biological Extracellular Vesicles, Antitumoral Drug Delivery, Dictyostelium discoideum

\section{Introduction}

The cell is the factory of most primitive unicellular organisms, the building block of plants and animals, as well as of human tissues. In higher eukaryotic organisms, cells are protected by their membrane, from xenobiotics, including many therapeutic drugs. Life dysfunctions, involved in human diseases and cancers, are often to be corrected inside the cell, at different vital targets, like nucleus, mitochondria, Golgi.... Both the old therapies, like antitumoral chemotherapies, and the new ones, like gene therapies, are faced with the major problem of efficient drug delivery. Therefore, the goal of all the drug delivery strategies will be to design Trojan horses to pass the lipid membrane barrier without destroying it and safely convey therapeutic drugs to their cellular targets [1].

Many different Trojan horse strategies for drug delivery have already been designed [2], like using the viral capsides and engineered lipid-like or polymeric vesicles, as well as many new associated technologies [2-4].

A search for the mechanisms, elicited by the nonpathogenic unicellular eukaryotic microorganism Dictyostelium discoideum to get rid of many structurally unrelated xenobiotics lead us to elaborate a different bi- ological Trojan horse strategy.

First, we found that these cells "made the difference" between benzo(a)pyrene, $\mathrm{B}(\mathrm{a}) \mathrm{P}$, the main carcinogenic compound of tobacco smoke, and its non-carcinogenic isomer benzo(e)pyrene, B(e)P. Namely, B(a)P was ejected from the cells, whereas $\mathrm{B}(\mathrm{e}) \mathrm{P}$ could remain into the cells [5]. In the early 1990s, when antitumoral multidrug resistance was thought to be mainly mediated by the P-glycoprotein $\mathrm{ABC}$-transporter, we wondered whether the P-glycoprotein might mediate the $\mathrm{B}(\mathrm{a}) \mathrm{P}$ efflux from the $D$. discoideum cells. Indeed, we evidenced the presence of this human-like transporter in $D$. discoideum cells [6]. However, it was shown to be inefficient for their drug detoxification.

In quite different cell synchronization experiments, we noticed that the widely-used vital DNA-specific stain, Hoechst 33342 (HO342) was unable to stain the nuclei of $D$. discoideum cells. When grown in the presence of HO342, the cell media harboured plenty of fluorescent microstructures, which turned out to be microvesicles, in holding the dye [7].

After studying their characteristics, we discovered that these biologically elaborated nanovesicles, ejected in the extracellular medium, were good candidates for "tricking" the known difficult entry of therapeutic drugs into 
human cells. Using HO342, we have shown that the isolated dye-containing vesicles were able to deliver it into the nuclei of naive Dictyostelium cells, thus overcoming their constitutive resistance to the free dye. Moreover, with living human leukemia multidrug resistant cells, K562r, known to be resistant to HO342, a Dictyostelium vesicle-mediated dye-transfer into the nuclei was also evidenced [8]. Control experiments showed that Dictyostelium vesicle-release was not a mere detoxification mechanism, but was both constitutive (Tatischeff, et al., 1998) and important for intercellular communication.

With the goal of using these biological extracellular nanovesicles for drug delivery, an in vitro investigation was conducted on HeLa cells, with hypericin, a hydrophobic fluorescent photosensitizer, aimed for antitumoral photodynamic therapy. Thus, we found that Dictyostelium nanovesicles, biologically loaded with this therapeutic molecule, are a promising nanodevice for cellular drug delivery [9].

In the present paper, we intend to further propose the use of $D$. discoideum extracellular nanovesicles for drug delivery ${ }^{1}$. We summarize their main characteristics, and stress their efficiency for drug delivery into tumoral human cells, as shown by our in vitro experiments. The advantages upon the use of these nanovesicles, when compared with other cell-derived microvesicles are discussed.

\section{Materials and Methods}

All the materials and methods used in our work on Dictyostelium nanovesicles have been detailed in our previous papers [7-9]. Only the methods dealing with Dictyostelium cell cultures and preparation of vesicles will be recalled here for clarity.

\subsection{Cell Culture}

Dictyostelium cells, Ax-2 strain, were grown in suspension in the dark, on a gyratory shaker $(150 \mathrm{rpm})$ at $+22^{\circ} \mathrm{C}$, in HL5 semi-defined medium [10], containing penicillin $(50 \mathrm{U} / \mathrm{mL})$ and streptomycin $(50 \mathrm{U} / \mathrm{mL})$ (Biomedia, Boussens, France). In contrast with mammalian cells, Dictyostelium cells are grown without fetal calf serum. For proper oxygenation, each suspension was grown in an Erlenmeyer containing five times the suspension volume.

\subsection{Preparation of Dictyostelium Nanovesicles}

Dictyostelium cell cultures, were initiated at $5 \times 10^{5}-10^{6}$

\footnotetext{
${ }^{1}$ Tatischeff, I., Alfsen, A., Lavialle, F. "Extracellular vesicles from non-pathogenic amoebae useful as vehicle for transferring a molecule of interest to an eukaryotic cell" Patent european priority N 03291 752 07/15/2003 (DRITT-UPMC) (European Patents in Danemark, Deutschland, France, Great Britain, Italy, Netherland and Spain), US Patent and Pending Canadian Patent).
}

cells $\cdot \mathrm{ml}^{-1}$ and grown in suspension in HL5 medium in the absence (control) or in the presence of the drug to be vectorized. For preparation of nanovesicles, cells were generally used in the late exponential or early stationary phase of growth, at a cell density about $10^{7}$ cells $\cdot \mathrm{ml}^{-1}$. One has to check that the drug does not affect cell growth. After $48 \mathrm{~h}$ of growth, both cell suspensions were centrifuged at $700 \times g$ for $5 \mathrm{~min}\left(+20^{\circ} \mathrm{C}\right)$. The cell-free media were centrifuged at $2000 \times \mathrm{g}$ for $10 \mathrm{~min}\left(+20^{\circ} \mathrm{C}\right)$. The $2000 \times \mathrm{g}$ supernatants were centrifuged at $12,000 \mathrm{x} g$ for $30 \mathrm{~min}\left(+4^{\circ} \mathrm{C}\right)$. Nanovesicles present in the pellets were concentrated $(\times 100)$ in phosphate buffered saline (PBS), pH 7.2 without calcium and magnesium (GIBCO).

These nanovesicles were quite stable in PBS, as they did withstand repeated liquid nitrogen freeze-thaw cycles without breaking. As observed with nanovesicles in holding $\mathrm{HO} 342$, they could be kept at $+4^{\circ} \mathrm{C}$ at least 2 months without releasing the dye.

\section{Main Results}

In the Eukarya branch of the evolutionary tree, Dictyostelium discoideum (http://www.dictybase.org), an ancestral non-pathogenic amoeba, placed at the border between the plant and animal kingdoms, is equipped with a lipid membrane that plays a critical role in many aspects of cell development. As in other eukaryotic cells, Dictyostelium plasma membrane contains proteins, lipids and carbohydrates. The phospholipid content of the plasma membrane of axenically grown vegetative Ax-2 cells is not very different from the one of mammalian cell membranes, except for an extremely high amount of unsaturated fatty acids, making up $75 \%-90 \%$ of the fatty acids of the organism and of the membrane, respectively. The membrane sterol is stigmasterol, instead of the mammalian cholesterol.

\subsection{Characteristics of Dictyostelium Nanovesicles}

Morphological analysis of control vesicles prepared from the cell growth medium was carried out by cryoelectron microscopy imaging. The vesicles appeared mostly smooth and rounded, delineated by a lipid membrane bilayer (Figure 1). The histogram of their heterogeneous size distribution indicated that almost $80 \%$ of them have an average diameter within a range of $50-150 \mathrm{~nm}$ [9]. $\mathrm{CdSe} / \mathrm{CdZnS}$ quantum dots phospholipid micelles were used, to check whether Dictyostelium cells might be detoxified from these quantum dots by following the vesicle-mediated pathway. As shown on Figure 1, contrary to many xenobiotics, these quantum dots were excreted from Dictyostelium cells, as aggregates outside the vesicles. This observation indicates that all the foreign compounds, incubated with Dictyostelium cells, are not deto- 


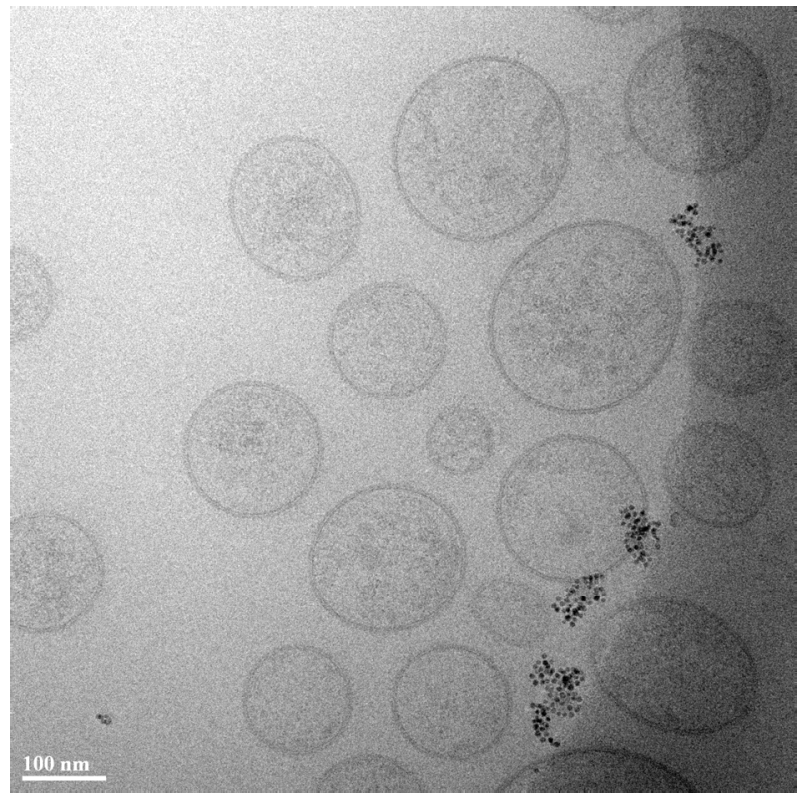

Figure 1. Morphological characterization of Dictyostelium nanovesicles by cryoelectron microscopy. For methodological details, cf. [9]. As shown on the figure, CdSe/CdZnS quantum dots phospholipid micelles, incubated with growing Dictyostelium cells, were excreted as (black) aggregates outside the nanovesicles.

xified by means of the extravesicular pathway. Further ongoing studies should help understanding the structural characteristics required for a drug vesicle-mediated transport.

A proteomic analysis of the control vesicles revealed a predominance of actin and actin-related proteins. The detection of a lysosomal membrane protein (LIMP II) [9] indicated that these vesicles are likely generated in the late endosomal compartment, as the known exosomes of many mammalian cells.

Lipid analysis of the bilayer surrounding the vesicles [7] showed, beside the phospholipids and lipids common in the plasma membrane: PC, PI, PS and PE, sphingomyelin, PG and DPG, the presence of lyso bis-phosphatidic acid (LBPA), a lipid inducing membrane fusion [11]. Noteworthy, this unusual phospholipid is a major membrane constituent of the internal vesicles of multivesicular bodies [12-14], like the known exosomes of many human cells. These observations strengthen the hypothesis of a multivesicular origin for the nanovesicles released by Dictyostelium cells and emphasize the role of the nature of the lipids in the formation and function of Dictyostelium vesicles.

\subsection{Vesicle-Mediated Transfer of Hoechst 33342 to the Nuclei of Human Leukemia Resistant Cells, K562r}

HO342-loaded nanovesicles allowed to label the nuclei of naive Dictyostelium cells, thus overcoming their con- stitutive resistance to the free dye [8]. The ability of these nanovesicles, to transfer $\mathrm{HO} 342$ to the nuclei of human leukemia living cells, was investigated by adding the nanovesicles $(\times 50)$ to multidrug resistant $\mathrm{K} 562 \mathrm{r}$ cells (Figure 2). Four cells were observed by phase contrast microscopy after about $4 \mathrm{~h}$ incubation (Figures 2(a), (c), (e) and (g)). The efficient transfer of HO342 to the cell nuclei was observed under UV excitation. The simultaneous observation of the same cells with white light and UV excitation (Figures 2(b), (d), (f) and (h)) clearly showed that the HO342-stained nuclei were inside the K562r cells. Thus, the Dictyostelium nanovesicle-mediated HO342 delivery had overcome the induced resistance of K562r cells to the vital staining of their nuclei.

\subsection{Vesicle-Mediated Transfer of Hypericin to the Golgi of Human Cervix Carcinoma HeLa Cells}

Hypericin transfer to target cells was first visualized using human fibroblasts (HS68) as control cells and with HeLa cells used as a model of tumoral cells. After a 1-h incubation in the presence of hypericin packaged into nanovesicles, a fluorescent signal was detected almost exclusively in the perinuclear area of HeLa cells (Figure 3). Under these experimental conditions, neither the cell plasma membrane, nor the nucleus, was labeled by the hydrophobic cargo molecule.

Similar experiments were conducted using free hypericin, i.e. not encapsulated into vesicles, for comparison. After only a 30-min incubation, HeLa cells became round-shaped, the typical morphology of dying cells that have lost their adhesion properties (Figure 3). This result demonstrates that the nanovesicles vectorize significant amounts of drug, but also prevent the uncontrolled cell
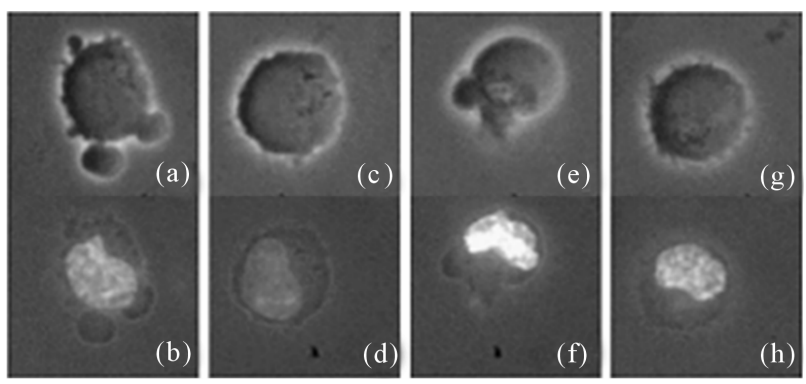

Figure 2. Transfer of HO342 mediated by the Dictyostelium nanovesicles to the nuclei of living human leukaemia cells, K562r. Cells were incubated (v/v) with these nanovesicles $(\times 50)$. Four different K562r cells are shown, in the range of incubation time $3 \mathrm{~h} 40-4 \mathrm{~h} \mathrm{10}$, as observed either with phase contrast ( $\times 40 \mathrm{CP}$ objective) (a, c, e, g), or with both white light and UV fluorescence microscopy $(\times 40 \mathrm{UV}$ objective) (b, d, f, h). Figure reproduced with permission from [8]. 


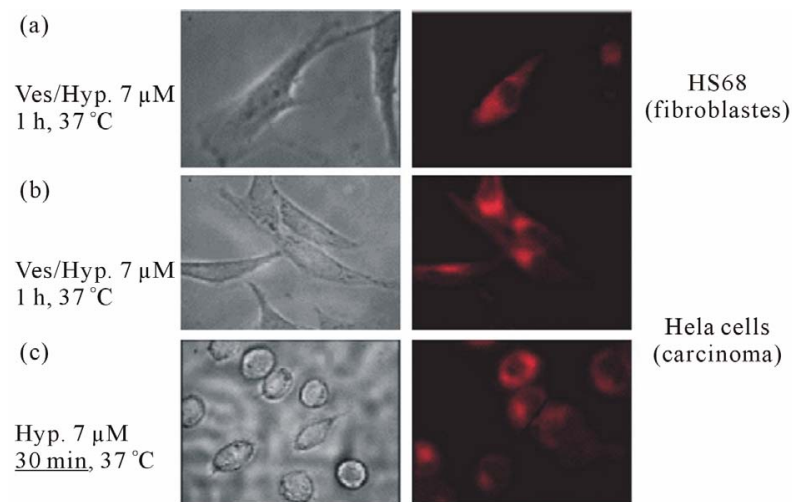

Figure 3. Light-transmission and fluorescence microscopy of hypericin internalization within human target cells. Human fibroblasts (HS68) (a) and HeLa cells (b and c) were incubated at $37^{\circ} \mathrm{C}$ in the dark for the indicated times, in the presence of $7 \mu \mathrm{M}$ hypericin packaged within Dictyostelium nanovesicles (a and b) or free in DMEM (c). Figure reproduced with permission from [9].

death triggered by free hypericin under the blue light used for observation.

To check the intracellular localization of hypericin delivered by the nanovesicles into HeLa cells, co-localization studies were performed with fluorescent markers of the Golgi apparatus (BODIPY ceramide) and of lysosomes (LysoTracker). Within $1 \mathrm{~h}$, hypericin was shown to coaccumulate with BODIPY into the Golgi apparatus, and the hypericin-loaded nanovesicles were efficient for photodynamic killing of HeLa cells [9].

The previous studies were initiated to investigate the potential use of Dictyostelium cell-released nanovesicles as in vitro drug carriers for cancer therapy. Dictyostelium cells were able to release into the culture medium nanovesicles loaded either with $\mathrm{HO} 342$ or with hypericin, a photosensitizer differing in charge and hydrophobicity from the DNA marker. Additionally, these nanovesicles were shown to respectively label human leukaemic resistant cells K562r, and human cervix carcinoma, HeLa, cells. It is worth stressing that the two cargo molecules, HO342 and hypericin, were transferred to two distinct intracellular locations, namely, the nucleus for HO342 and the Golgi apparatus for hypericin.

Vesicular-mediated delivery of hypericin depicted good photo efficiency for killing HeLa cells. Using vesicular hypericin, instead of free hypericin, ensures a better control of drug cell loading, by an easy manipulation of the amount of vesicles incubated with the cells. Most importantly, the vesicular delivery prevents the detrimental aggregation of free hypericin in aqueous media.

\section{Discussion}

Cell membranes are a structured self-assembly of lipids interacting with proteins and in contact with both exter- nal medium and the internal aqueous solvent and solutes. They constantly form lipid vesicles that either move internally from the cell membrane to sites within the cell or are externalized. These vesicles are formed of a selection of membrane lipids and are characterized by specific properties, either of fusion and/or of targeting, that give them their dynamic properties, allowing the exchange of materials and informations.

From prokaryotic unicellular organisms to primitive eukaryotes, at the border between the vegetal and animal kingdoms, like the amoebae Dictyostelium discoideum up to mammalian cells [15] the formation of extracellular vesicles of different sizes, origins and functions, was shown to be a property of almost all cell types. Thus, extracellular vesicles have been described as originating from almost all mammalian cell types: human blood cells (platelets [16], B lymphocytes [15], dendritic cells [17], but also from intestinal epithelial cells [18] or from different pathological cells [15].

All these extracellular vesicles are examples of "vesicles shipping extracellular messages" [19] and "triggering intercellular communication" from one cell to another, either by fusion with the target cell [20] and/or by allowing internalization of signaling molecules [15,21]. These extracellular vesicles are, therefore, also potential Trojan Horses for drug delivery to target cells.

The exosomes, originating from the endosomal traffic [22], are mostly considered as candidates to develop genetic vaccines for immunotherapy [23], but have recently been also considered for drug delivery [24]. However, as mentioned by these authors, many "critical obstacles to the clinical translation of exosome-based therapy" remain. The growth of Dictyostelium cells in a semi-defined medium, without any serum, as compared to more complex human cell media, might favor this simple eukaryotic cell model for solving some of the remaining problems. Moreover, it is to be stressed, that our drug delivery device, based on a detoxification process, includes the Dictyostelium cell conditioning of the therapeutic drug into the vesicles that will be released. Therefore, no drug internalization agents into the vesicles are needed, as the ones in exosome-based therapy. Bacterially derived particles have also to be further loaded with therapeutic agents, in order to be efficient carriers [25]. Therefore, among all the cell-derived extracellular vesicles, Dictyostelium nanovesicles present the unique advantage of being cell-engineered for drug delivery.

With regard to a potential strong immunogenicity of these nanovesicles, due to their microbial origin, which might be a serious drawback for their use in vivo, it has already been documented for bacterial derived particles. By contrast, a first in vivo study upon the immunogenicity of Dictyostelium nanovesicles, intravenously injected 
twice in the tail of Balb/C mice, has shown a specific antibody response, but no pyrogenic response nor any inflammation, as measured by five pertinent cytokines (study performed by Genosafe, Evry, France). This should minimize the risk of undesirable immune responses upon in vivo administration of these biological nanovesicles, such as those triggered by tumor-released exosomes [26].

\section{Conclusions}

Our work indicates that $D$. discoideum is a bioengineering designer able to formulate vesicular drug carriers. To our knowledge, this is the first study describing cell-engineered vesicles able to both load a therapeutic molecule and vectorize it into human cells, as we have shown with the important antitumoral photosensitizer, hypericin [9].

Other characteristics of Dictyostelium vesicles appear interesting for targeting purpose; namely, the presence of discoidins I and II [9], which bind to the cell membrane with a specificity for galactose-related residues, might be of interest for lectin-mediated drug delivery [27]. The targeting strategy, tested with bacterially derived vesicles [25], by using bi-specific antibodies, with one arm recognizing a component of the vesicle surface, the other a cell surface receptor of the target cell, might also be applied to Dictyostelium nanovesicles. Another vectorization strategy could be developed by using Dictyostelium cells cultured in the presence of magnetic nanoparticles to engineer magnetic nanovesicles [28].

With regard to the possibility of using Dictyostelium nanovesicles in therapy, many problems remain to be solved. A study to elucidate the membrane events involved in cell entry of the drug vectorized by the nanovesicles should be performed. For further in vivo approaches, toxicity and allergenic properties of more purified nanovesicle preparations are to be characterized. Taking into account the described culture of Dictyostelium cells in bioreactors [29], a drug vectorization strategy could, indeed, be developed at a large pharmacological scale.

\section{Acknowledgements}

Eric Larquet (CNRS, UMR7590, UPMC, IMPMC, Paris, France) is kindly acknowledged for the cryoelectron microscopy measurements and Benoit Dubertret (CNRS UPR A0005, UPMC, ESPCI, Paris, France) for the gift of the $\mathrm{CdSe} / \mathrm{CdZnS}$ quantum dots.

\section{REFERENCES}

[1] W. M. Pardridge, "Molecular Trojan Horses for BloodBrain Barrier Drug Delivery," Current Opinion in Pharmacology, Vol. 6, No. 5, 2006, pp. 494-500. doi:10.1016/j.coph.2006.06.001

[2] Y. Seow and M. J. Wood, "Biological Gene Delivery Vehicles: Beyond Viral Vectors," The American Society of Gene Therapy, Vol. 17, No. 5, 2009, pp. 767-777.

[3] K. M. Kroeger, A. K. M. G. Muhammad, G. J. Baker, H. Assi, M. K. Wibowo, W. Xiong, K. Yagiz, M. Candolfi, P. R. Lowenstein and M. G. Castro, "Gene Therapy and Virotherapy: Novel Therapeutic Approaches for Brain Tumors," Discovery Medicine, Vol. 10, No. 53, 2010, pp. 293-304.

[4] A. Villaverde, "Nanotechnology, Bionanotechnology and Microbial Cell Factories," Microbial Cell Factories, Vol. 9, 2010, pp. 53-56. doi:10.1186/1475-2859-9-53

[5] I. Tatischeff, F. Lavialle, C. de Paillerets, H. Weintraub, G. Tham and A. Alfsen, "Carcinogenic Benzo(a)pyrene and Non-Carcinogenic Benzo(e)pyrene Discriminated by Dictyostelium discoideum Cells through Internalization," In: P. Garrigues and M. Lamotte, Eds., Polycyclic Aromatic Compounds, PAH XIII, Gordon and Breach Science Publishers, Switzerland, 1993, pp. 695-702.

[6] I. Tatischeff and F. Lavialle, "Immunological Evidence of a P-Glycoprotein in the Microorganism Dictyostelium," Comptes Rendus de l'Académie des Sciences III (Paris), Vol. 316, No. 6, 1993, pp. 560-563.

[7] I. Tatischeff, M. Bomsel, C. de Paillerets, H. Durand, B. Geny, D. Segretain, E. Turpin and A. Alfsen, "Dictyostelium discoideum Cells Shed Vesicles with Associated DNA and Vital Stain Hoechst 33342," Cellular and Molecular Life Sciences, Vol. 54, No. 5, 1998, pp. 476-487. doi:10.1007/s000180050176

[8] I. Tatischeff, F. Lavialle, S. Pigaglio-Deshayes, C. Péchoux-Longin, L. Chinsky and A. Alfsen, "Dictyostelium Extracellular Vesicles Containing Hoechst 33342 Transfer the Dye into the Nuclei of Living Cells: A Fluorescence Study," Journal of Fluorescence, Vol. 18, No. 2, 2008, pp. 319-328. doi:10.1007/s10895-007-0271-4

[9] F. Lavialle, S. Deshayes, F. Gonnet, E. Larquet, S. G. Kruglik, N. Boisset, R. Daniel, A. Alfsen and I. Tatischeff, "Nanovesicles Released by Dictyostelium Cells: A Potential Carrier for Drug Delivery," International Journal of Pharmaceutics, Vol. 380, No. 1-2, 2009, pp. 206-215. doi:10.1016/j.ijpharm.2009.06.039

[10] D. J. Watts and M. Ashworth, "Growth of Myxameobae of the Cellular Slime Mould Dictyostelium discoideum in Axenic Culture," Biochemical Journal, Vol. 119, No. 2, 1970, pp. 71-174.

[11] J. Gruenberg, "Lipids in Endocytic Membrane Transport and Sorting," Current Opinion in Cell Biology, Vol. 15, No. 4, 2003, pp. 382-388. doi:10.1016/S0955-0674(03)00078-4

[12] T. Kobayashi, K. Startchev, A. J. Whitney and J. Gruenberg, "Localization of Lysobisphosphatidic Acid-Rich Membrane Domains in Late Endosomes," Biological Chemistry, Vol. 382, No. 3, 2001, pp. 483-485. doi:10.1515/BC.2001.059

[13] T. Kobayashi, M. H. Beuchat, J. Chevallier, A. Makino, N. Mayran, J. M. Escola, C. Lebrand, P. Cosson, T. Ko- 
bayashi and J. Gruenberg, "Separation and Characterization of Late Endosomal Membrane Domains," Journal of Biological Chemistry, Vol. 277, No. 35, 2002, pp. 3215732164. doi:10.1074/jbc.M202838200

[14] C. Yorikawa, H. Shibata, S. Waguri, K. Hatta, M. Horii, K. Katoh, T. Kobayashi, Y. Uchiyama and M. Maki, "Human CHMP6, a Myristoylated ESCRT-III Protein, Interacts Directly with an ESCRT-II Component EAP20 and Regulates Endosomal Cargo Sorting," Biochemical Journal, Vol. 387, No. 1, 2005, pp. 17-26. doi:10.1042/BJ20041227

[15] B. Hugel, M. C. Martínez, C. Kunzelmann and J. M. Freyssinet, "Membrane Microparticles: Two Sides of the Coin," Physiology, Vol. 20, 2005, pp. 22-27. doi:10.1152/physiol.00029.2004

[16] H. F. G. Heijnen, A. E. Schiel, R. Fijnheer, H. J. Geuze and J. J. Sixma, "Activated Platelets Release Two Types of Membrane Vesicles: Microvesicles by Surface Shedding and Exosomes Derived from Exocytosis of Multivesicular Bodies and Alpha-Granules," Blood, Vol. 94, No. 11, 1999, pp. 3791-3799.

[17] K. Denzer, M. van Eijk, M. J. Kleijmeer, E. Akobson, C. de Groot and H. J. Geuze, "Follicular Dendritic Cells Carry MHC Class II-Expressing Microvesicles at their Surface," The Journal of Immunology, Vol. 165, No. 3, 2000, pp. 1259-1265.

[18] G. Van Niel, G. Raposo, C. Candalh, M. Boussac, R. Hershberg, N. Cerf-Bensussan and M. Heyman, "Intestinal Epithelial Cells Secrete Exosome-Like Vesicles," Gastroenterology, Vol. 121, No. 2, 2001, pp. 337-349. doi:10.1053/gast.2001.26263

[19] B. Fevrier and G. Raposo, "Exosomes: Endosomal-Derived Vesicles Shipping Extracellular Messages," Current Opinion in Cell Biology, Vol. 16, No. 4, 2004, pp. 415421. doi:10.1016/j.ceb.2004.06.003

[20] L. Chernomordik, M. M. Kozlov and J. Zimmerberg, "Lipids in Biological Membrane Fusion," The Journal of Membrane Biology, Vol. 146, No.1, 1995, pp. 1-14. doi:10.1007/BF00232676

[21] M. P. Wymann and R. Schneiter, "Lipid Signalling in Disease," Nature Reviews Molecular Cell Biology, Vol. 9, No. 2, 2008, pp. 162-176. doi:10.1038/nrm2335
[22] K. Denzer, M. J. Kleijmeer, H. F. G. Heijnen, W. Stoorvogel and H. J. Geuze, "Exosome: From Internal Vesicle of the Multivesicular Body to Intercellular Signaling Device," Journal of Cell Science, Vol. 113, No. 19, 2000, pp. 3365-3374.

[23] A. Delcayre and J. B. Le Pecq, "Exosomes as Novel Therapeutic Nanodevices," Current Opinion in Molecular Therapy, Vol. 8, No. 1, 2006, pp. 31-38.

[24] S. Lakhal and M. J. Wood, "Exosome Nanotechnology: An Emerging Paradigm Shift in Drug Delivery: Exploitation of Exosome Nanovesicles for Systemic in Vivo Delivery of RNAi Heralds New Horizons for Drug Delivery across Biological Barriers," Bioessays, Vol. 33, No. 10, 2011, pp. 737-741. doi:10.1002/bies.201100076

[25] J. A. MacDiarmid, N. B. Mugridge, J. C. Weiss, L. Phillips, A. L. Burn, R. P. Paulin, J. E. Haasdyk, K. A. Dickson, V. N. Brahmbhatt, S. T. Pattison, A. C. James, G. Al Bakri, R. C. Straw, B. Stillman, R. M. Graham and H. Brahmbhatt, "Bacterially Derived $400 \mathrm{~nm}$ Particles for Encapsulation and Cancer Cell Targeting of Chemotherapeutics," Cancer Cell, Vol. 11, No. 5, 2007, pp. 431-445. doi:10.1016/j.ccr.2007.03.012

[26] R. Valenti, V. Huber, M. Iero, P. Filipazzi, G. Parmiani and L. Rivoltini, "Tumor-Released Microvesicles as Vehicles of Immunosuppression," Cancer Research, Vol. 67, No. 7, 2007, pp. 2912-2915. doi:10.1158/0008-5472.CAN-07-0520

[27] C. Bies, C. M. Lehr and J. F. Woodley, "Lectin-Mediated Drug Targeting: History and Applications," Advanced Drug Delivery Reviews, Vol. 56, No. 4, 2004, pp. 425435. doi:10.1016/j.addr.2003.10.030

[28] C. Wilhelm, F. Lavialle, C. Péchoux, I. Tatischeff and F. Gazeau, "Intracellular Trafficking of Magnetic Nanoparticles to Design Multifunctional Biovesicles," Small, Vol. 4, No. 5, 2008, pp. 577-582. doi:10.1002/smll.200700523

[29] Y. Lu, J. C. Knol, M. H. K. Linskens, K. Friehs, P. J. M. Van Haastert and E. Flaschel, "Production of the Soluble Human Fas Ligand by Dictyostelium discoideum Cultivated on a Synthetic Medium," Journal of Biotechnology, Vol. 108, No. 3, 2004, pp. 243-251. doi:10.1016/i.jbiotec.2003.12.006 